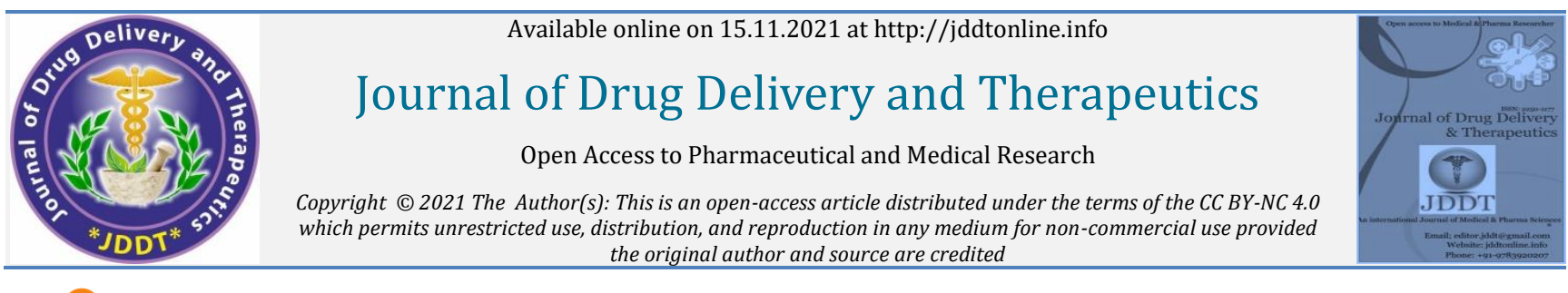

Open Access Full Text Article

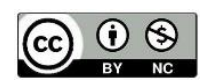

Review Article

\title{
How does Heparan Sulfate and COVID-19 Work?: An Overview
}

\author{
Alby Anna ${ }^{1}$, DSambath Kumar R ${ }^{2 *}$, (D) Venkateswaramurthy $\mathrm{N}^{3}$ \\ ${ }^{1}$ Post Graduate Student, Department of Pharmacy Practice, J.K.K. Nattraja College of Pharmacy, Namakkal (Dt), Kumarapalayam- 638 183, Tamil \\ Nadu, India. \\ ${ }_{2}^{2}$ Professor and Head, Department of Pharmaceutics, J.K.K. Nattraja College of Pharmacy, Namakkal (Dt), Kumarapalayam- 638 183, Tamil Nadu,
} India.

${ }^{3}$ Professor and Head, Department of Pharmacy Practice, J.K.K. Nattraja College of Pharmacy, Namakkal (Dt), Kumarapalayam- 638 183, Tamil Nadu, India.

\section{Article Info:}

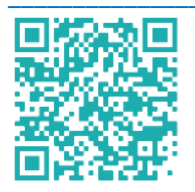

\section{Article History:}

Received 23 September 2021

Reviewed 22 October 2021

Accepted 27 October 2021

Published 15 November 2021

\section{Cite this article as:}

Anna A, Sambath Kumar R, Venkateswaramurthy N, How does Heparan Sulfate and COVID-19 Work?: An Overview, Journal of Drug Delivery and Therapeutics. 2021; 11(6):244-247

DOI: http://dx.doi.org/10.22270/jddt.v11i6.5138

\section{*Address for Correspondence:}

Dr. R. Sambath Kumar, Professor and Head Department of pharmaceutics, J.K.K. Nattraja College of Pharmacy, Kumarapalayam-638183, Tamil Nadu, India.

ORCID ID: https://orcid.org/0000-0003-1454-9582

\section{Abstract}

Globally, the severe acute respiratory syndrome coronavirus-2 (SARS-CoV-2) had infected over 3 million individuals and claimed many lives producing a global epidemic that necessitates the rapid development of therapeutic solutions. The ideal technique for quickly deploying well-characterized medicines against novel infections is known as drug repurposing. Several repurposable medicines are currently being tested to see if they may be used to treat COVID-19. Heparin, which is commonly utilized to reduce thrombotic events associated with COVID-19-induced disease, is one such promising drug. Heparansulphate is prevalently expressed in mammalian tissues. CoV-2 requires the helping cofactor heparansulphate (HS) on the cell surface: knocking down genes related in HS formation or treating cells with an HS mimic both prevent spike-mediated viral entrance. Heparin/HS binds directly to spike and promotes viral entrance by facilitating the attachment of spikebearing viral particles to the cell surface. As documented with cell surface-bound heparansulphate, heparin binding to the open conformation of the spike structurally supports the state and may enhance ACE2 binding. Thus, heparansulphate could potentially be utilised to prevent SARS-CoV-2 transmission, based on available datas also consumption of heparansulphate during SARS-CoV-2 cellular entrance may play a role in the thrombotic events associated with COVID-19 infection. Furthermore, this study provides the findings on the mechanism(s) by which heparansulphate could slow the progression of SARS-CoV-2 infection.

Keywords: COVID-19, HeparanSulphate, Spike Protiens

\section{INTRODUCTION:}

COVID-19 (SARS CoV2), a lethal virus that causes severe acute respiratory syndrome (ARDS) and hospital pneumonitis, is currently sweeping the globe. The virus was first discovered in a series of cases in Wuhan, China, and the World Health Organization (WHO) termed it the coronavirus disease 2019 (COVID-19) pandemic due to its rapid spread 1,2. The SARS-CoV-2 pandemic necessitates the development of therapeutic strategies shortly. COVID-19 patients have clotting problems, which have a negative impact on the disease's prognosis and lead to greater fatality rates 3-5. Abnormal coagulation indicators, especially notably raised $\mathrm{d}$ dimer, fibrin degradation product (FDP), extended prothrombin time, and thrombocytopenia, have been found in severe COVID-19 patients and non-survivors in numerous studies 6,7. Overt disseminated intravascular coagulation (DIC) is more common in patients infected with this coronavirus ${ }^{7-9}$. The Heparansulphate proteoglycan (HSPG) and Heparanase system is one of the probable systems that may play a key role in the excessive coagulation that characterises COVID-19 1 .
The binding of a viral protein to the cell surface heparansulphate (HS) is usually the first of several interactions that lead to viral entry and infection. Selectins and cytokines (e.g., IL-6 and TNF-) interact with HS expressed on endothelial cells to limit immune cell recruitment during inflammation, on the other hand 7 . Endothelial barrier function is required for the regulation of fluid and protein extravasation, particularly in the lungs and kidneys ${ }^{10}$. Endothelial cell dysfunction appears to have a key part in the pathogenesis of COVID-19 issues, according to several studies ${ }^{11,12}$.

The glycocalyx is a thick layer of negatively charged glycosaminoglycans (GAGs) that covers endothelial cells. Heparansulphate (HS) is the most frequent sulfated GAG in the glycocalyx. HS contributes to the endothelium's chargedependent barrier function due to its negative charge ${ }^{13}$. Heparanase (HPSE), the only known mammalian HS degrading enzyme, degrades the endothelium glycocalyx, causing endothelial barrier function to be lost, as shown in ARDS and proteinuric renal diseases 14,15 . In addition to altering barrier function, HPSE forms a pro-inflammatory 
glycocalyx that promotes the binding of chemokines, cytokines, and leukocytes to the endothelial cell surface ${ }^{16}$. HPSE inhibition and/or deficiency are useful in experimental lung and renal illnesses ${ }^{14,15}$. Heparins, particularly low molecular weight heparins (LMWH), are potent inhibitors of HPSE activity and have been proposed as a treatment for COVID-19 patients. ${ }^{17}$

\section{WHAT IS HEPARAN SULFATE?}

On the cell surface and in the extracellular matrix, HSPGs are abundant (ECM). Proteins, hormones, cytokines, and growth factors bind to binding sites on the cell surface, where they perform crucial activities in cell-ECM interactions.18 Heparansulphate (HS) sequences are partially sulfated carbohydrate portions of proteoglycans found on nearly every human cell surface. The saccharide chains in HS chains are slightly different from those in heparin. They're made up of D-glucosamine and glucuronic acids in that order (Liduronic and Dglucuronic acids). In the intracellular milieu and extracellular matrix, HS interacts with a variety of soluble and insoluble ligands, including cytokines and growth hormones. ${ }^{19}$ The HS and surface proteins are also used by many bacteria, viruses, and poisons to bind to human cells. The major mechanism for this binding is charge, or electrostatic contact, with the negatively charged HS attracting the basic amino acids of surface proteins.[18]The functions of heparin and HS are mediated via interactions with a range of proteins, including proteases, protease inhibitors, chemokines, cytokines, growth factors, and their receptors, all of which have variable degrees of specificity. ${ }^{20}$

\section{HEPARAN SULFATE IN VIRUSES:}

HSPGs serve as a binding receptor for several human viruses, including coronaviruses 21,22 , making them a target of many investigations aimed at limiting this early interaction and thereby preventing viral entry 23,24. Many viruses use HS as a key attachment co-receptor, including herpes simplex virus (HSV), respiratory syncytial virus (RSV), dengue virus (DENV), varicella zoster virus, human immunodeficiency virus (HIV), Epstein-Barr virus, vaccinia virus, hepatitis C virus, and others. During the present COVID-19 outbreak, direct interaction of HS proteoglycans with co-receptor Angiotensin-converting enzyme 2 (ACE2) is critical for the attachment of spike-bearing viral particles of SARS-CoV and SARS-CoV-2 to the cell surface to promote viral entry. ${ }^{25}$ Heparin and other HS mimetics have been studied extensively and have been proven to inhibit infectivity and cell-to-cell transmission in a variety of viruses, including SARS-CoV-2. ${ }^{26}$

Several viruses exploit HSPG-mediated endocytosis to enter host cells 27 . Electrostatic interactions between negatively charged sulfated HS chains and basic residues of surface or capsid proteins are used by some viruses to increase their concentration at the host cell surface, hence increasing their binding to specific entrance receptors. ${ }^{21}$ Clathrin-mediated uptake, or caveolae/cholesterol-dependent endocytosis, and macropinocytosis are two of the HSPG-regulated endocytic mechanisms used by the virus to enter host cells. ${ }^{28}$ Some viruses that do not require HSPG binding to adhere and infect host cells may gain HSPG dependency after intra-host or cell culture adaption. Several viruses, including rhinoviruses, Coxsackie virus B3, Sindbis virus, Ross River alphavirus, flavivirus tick-borne encephalitis virus, and others, have been shown to adapt in cell culture, resulting in increased ability to bind HS, a phenomenon that could provide the viruses with a selective advantage. ${ }^{21}$ Several SARS-CoV-2 mutations impacting the $S$ protein sequence have been discovered, but more research is needed to determine whether and how these mutations affect the $S$ protein's ability to bind HSPGs, and therefore on SARS-CoV-2 infection, tropism, immunity, and pathogenesis. ${ }^{30}$

\section{COVS AND IT'S INVASION:}

CoVs are enclosed, positive-sense singlestranded (up to $30,000 \mathrm{bp}$ ) RNA viruses that belong to the Nidovirales order of the Othocoronavirinae subfamily. Infection with the viruses can cause severe diseases in people and other animals, affecting the upper respiratory, gastrointestinal, and central neurological systems. 31 According to the International Committee on Virus Taxonomy's 10th Report on Virus Taxonomy, CoVs can be divided into four genera based on a systematic investigation of viral nucleic acid sequence: alpha, beta, gamma, and delta (ICTV). ${ }^{32}$ There are currently seven representative strains of human coronaviruses (HCoVs), including four low-pathogenic coronaviruses [HCoV-229E, HCoV-NL63 (alphacoronaviruses), HCoV-OC43, and HCoV-HKU1 (betacoronaviruses)], which cause mild respiratory diseases in humans, and three high-pathogenic strains, including HCoVs severe acute (beta-coronaviruses). CoVs share similar genome identities. ${ }^{33}$ Two huge overlapping open reading frames (ORFs) near the $5^{\prime}$-terminus of the genome, ORF $1 \mathrm{a}$ and ORF $1 \mathrm{~b}$, encode polyproteins $1 \mathrm{a}(\mathrm{pp} 1 \mathrm{a})$ and pp1b/1ab, respectively. Spike $(S)$, which defines all coronaviruses, Envelope (E), Membrane (M), and Nucleocapsid (N) are the four common structural proteins encoded by one-third of the genome at the $3^{\prime}$ terminus $(\mathrm{N}) .{ }^{34}$ The $\mathrm{S}$ protein is a trimeric class I fusion protein that protrudes from the surface of the virion and serves as a receptor, membrane fusion protein, virus entrance protein, and antibody neutralizer. 35 Because of its critical functions during viral infection, the $\mathrm{S}$ protein is a prominent target for the development of antiviral medications, antibodies, entrance inhibitors, and vaccines. 15

$\mathrm{CoV}$ infection is divided into two stages: initial attachment to a cell surface receptor and fusion of the $S$ protein with the host cell membrane, which allows the nucleocapsid to be transferred to the target cell. CoVs have long been known to interact with cell surface molecules to boost their affinity for specific receptors. HS proteoglycans (HSPGs) are found in nearly all mammalian cells and function as virus coreceptors. ${ }^{36}$ They may be able to bind to the surface proteins of CoVs, boost subsequent recognition of a secondary Receptor (ACE2/DPP4), and increase local HSPG levels to facilitate virus attachment and entry. According to studies, different HS compositions may influence virus tropism and HS co-receptors on the host cell surface, causing conformational changes in CoVs' S proteins and possibly the formation of a ternary complex. 15,37,38 These findings suggested that the HS-S protein interactions could be a focus for preventing viral infection.

\section{MECHANISM OF HEPARAN SULFATE IN SARS- COV-2:}

Most mammalian cell types have heparansulphate (HS), which is a linear polysaccharide present on cell surfaces and in the extracellular matrix. HS is used by a variety of viruses to bind to the host cell's surface. Viruses use the HS interaction to raise their concentration on the cell surface and their chances of finding a more specific entrance receptor. ${ }^{37}$ SARS-CoV-2 connects to heparin via its surface protein Spike 1 receptor-binding domain. ${ }^{38}$ Because many bacteria attach to HS or heparin, using these adhesion routes to "trap" bacteria and viruses and remove them from circulation via extracorporeal treatment co uld be a promising strategy. Heparin also has a direct antibacterial and antiviral impact. Heparin, which is released from mast 
cells and basophils as a result of tissue damage, reduces hepcidin expression and interrupts the iron availability of Mycobacterium tuberculosum. Heparin has also been shown to reduce the cytopathogenicity of human immunodeficiency viruses by $50 \%$ at a concentration of $4.7 \mathrm{~g} / \mathrm{mL} .^{39}$ In several viruses and all pathogenic amyloid proteins, heparin binding is a common characteristic. These pathogens employ this property to bind to and internaliseheparansulphate proteoglycans (HSPGs) on host cells. As a result, heparin and Oheparan sulphate therapy reduces infectivity by preventing pathogens from binding to HSPGs. ${ }^{38}$ The receptor-binding area of the novel SARS-CoV-2 virus's S1 subunit of spike glycoprotein interacts with heparin, according to new research. ${ }^{40}$ HSPGs (heparansulphate proteoglycans) are cell surface receptors involved in the cellular uptake of harmful amyloid proteins and viruses, notably the new coronavirus, SARS coronavirus 2. (SARS-CoV-2). Heparin and heparansulphate block these pathogens' binding to HSPGs and hence prevent cellular internalisation, but their anticoagulant properties have limited their usage in the treatment of viral infections. ${ }^{41}$

Heparin is a highly effective anticoagulant that has long been used in hospitals. In addition to their anti-coagulant activity, heparin and its derivatives have been studied for their antiinflammatory, antiviral, anti-angiogenesis, antineoplastic, and anti-metastatic properties. Heparin-binding peptides (HBPs) are non-anticoagulant medications that prevent heparin-binding pathogens from binding to heparin-binding peptides (HSPGs).The virus's transmembrane spike (S) glycoprotein binds to the host's angiotensin-converting enzyme 2 (ACE2). This allows SARS-CoV-2 to enter cells. ${ }^{2}$ SARSCoV is similar to SARS-CoV-2 in that it uses the same receptor. 3 SARS-spike CoV-2's protein is made up of two subunits: S1 is responsible for binding to the host receptor, while S2 is responsible for membrane fusion. $18,40,41$.

\section{APPLICATIONS OF HEPARAN SULFATE IN COVID-19:}

Exogenous and competitive heparin/HS mimetics could interfere with virus infection and/or reduce inflammatory responses by altering HS-protein interactions. In reality, COVID-19 patients frequently have hypercoagulopathy, which is treated with heparin/LMWH. Patients with severe COVID-19 who were hospitalised and treated with LMWH or fondaparinux (an ultra-low-molecular-weight heparin) had a better prognosis in terms of death, according to recent research. It's worth noting that heparin-induced thrombocytopenia (HIT) has been recorded in COVID-19 individuals who were given heparin. 42,43 In the heparin/LMWH therapy of COVID-2019, proper dosing and real-time monitoring of anti-Xa activity are critical. $44 \mathrm{~A}$ retrospective cohort research indicated that in the hospitalised COVID-19 treated with LMWH, IL-6 levels were dramatically lowered while the percentage of lymphocytes was remarkably enhanced in comparison to the non-LMWHtreated group, ${ }^{35}$ showing the drug's anti-inflammatory effectiveness. In addition to systemic administration, intranasal or inhalation administration of heparin/LMWH has been described for the treatment of lung illnesses and inhalation damage. ${ }^{7}$

\section{CONCLUSION:}

Despite its well-established anti-coagulant and antiinflammatory properties, heparin/potential HS's antiSARSCoV-2 activity was just recently hypothesised. Because HS/heparin mimetics are often easier to produce than real HS oligosaccharides, blocking these HS protein interactions with them is a viable technique for generating new therapies.
Efforts to develop new assays and molecular modelling tools to examine the interactions of HS mimetics with their target proteins have aided these research.HS mimetics have antiviral action against a variety of viruses that employ HS as an entrance receptor, including SARS-CoV-2, and many amphiphilic HS mimetics have virucidal activity due to their large lipophilic side chains disrupting viral lipid envelopes. At therapeutically relevant concentrations, PG545, in particular, has shown promise in vitro and in vivo action against SARS-CoV-2. Although in vitro experiments and some clinical data have provided promising evidence of heparin/HS (including their mimetics) and heparin/HSinteracting molecules as anti-SARS CoV-2 drugs, there is still debate about the structure specificities of the heparin/HS chains for their interaction with the $S$ protein.

\section{REFERENCES:}

1. Kinaneh S, Khamaysi I, Karram T, Hamoud S. Heparanase as a potential player in SARS-CoV-2 infection and induced coagulopathy. Biosci Rep.2021 41(7):BSR20210290. 10.1042/BSR20210290

2. Tandon, R., Sharp, J. S., Zhang, F., Pomin, V. H., Ashpole, N. M., Mitra, D., et al. Effective inhibition of SARS-CoV-2 entry by heparin and enoxaparin derivatives. BioRxiv. 2020:140236.10.1101/2020.06.08.140236

3. Zhou, B., She, J.Q., Wang, Y.D. and Ma, X.C. Venous thrombosis and arteriosclerosis obliterans of lower extremities in a very severe patient with 2019 novel coronavirus disease: a case report. J. Thromb. Thrombolys. 2020; 50:229232.10.1007/s11239-020-02084-w

4. Zhang, Y., Xiao, M., Zhang, S. et al. Coagulopathy and antiphospholipid antibodies in patients with Covid-19. N. Engl. J. Med. 2020; 382:e38. 10.1056/NEJMc2007575

5. Bikdeli, B., Madhavan, M.V., Jimenez, D. et al. COVID-19 and thrombotic or thromboembolic disease: implications for prevention, antithrombotictherapy, and follow-up: JACC Stateof-the-Art Review. J. Am. Coll. Cardiol. 2020; 75:29502973.10.1016/j.jacc.2020.04.031

6. Wang, Y.D., Zhang, S.P., Wei, Q.Z. et al. COVID-19 complicated with DIC: 2 cases report and literatures review. ZhonghuaXue Ye XueZaZhi. 2020; 41:245-247. 10.3760/cma.j.issn.02532727.2020.0001

7. Yu M, Zhang T, Zhang W, Sun Q, Li H, Li JP. Elucidating the Interactions Between Heparin/Heparan Sulfate and SARS-CoV2-Related Proteins-An Important Strategy for Developing Novel Therapeutics for the COVID-19 Pandemic. Front MolBiosci. 2021; 25(7):628551.10.3389/fmolb.2020.628551

8. Guan, W.J., Ni, Z.Y., Hu, Y. et al. Clinical characteristics of coronavirus disease 2019 in China. N. Engl. J. Med. 2020 382:1708-1720. 10.1056/NEJMoa2002032

9. Tang, N., Li, D., Wang, X. and Sun, Z. Abnormal coagulation parameters are associated with poor prognosis in patients with novel coronavirus pneumonia. J. Thromb. Haemost. 2020; 18:844-847.10.1111/jth.14768

10. Haraldsson B, Nyström J, Deen WM. Properties of the Glomerular Barrier and Mechanisms of Proteinuria. Physiol Rev.2008; 88(2):451-87.10.1152/physrev.00055.2006

11. Varga Z, Flammer AJ, Steiger P, Haberecker M, Andermatt R, Zinkernagel AS, et al. Endothelial cell infection and endotheliitis in COVID-19. Lancet. 2020; 395(10234):1417-8. 10.1016/S0140-6736(20)30937-5

12. Sardu C, Gambardella J, Morelli M, Wang X, Marfella R, Santulli G. Is COVID-19 an endothelial disease? Clinical and basic evidence.2020:2020040204.10.20944/preprints202004.0204.v 1

13. Goldberg R, Meirovitz A, Hirshoren N, Bulvik R, Binder A, Rubinstein AM, et al. Versatile role of heparanase in 
inflammation. Matrix Biol. 2013; 2(5):23440.10.3389/fonc.2019.00331

14. Kiyan Y, Tkachuk S, Kurselis K, Shushakova N, Stahl K, Dawodu $D$, et al.Heparanase-2 protects from LPS-mediated endothelial injury by inhibitingTLR4 signalling. Sci Rep. 2019; 9(1):13591.10.1038/s41598-019-50068-5

15. Schmidt EP, Yang Y, Janssen WJ, Gandjeva A, Perez MJ, Barthel L et al. The pulmonary endothelial glycocalyx regulates neutrophil adhesion and lung injury during experimental sepsis. Nat Med .2012; 18(8):1217-23.10.1038/nm.2843

16. Goldberg R, Meirovitz A, Hirshoren N, Bulvik R, Binder A, Rubinstein AM, et al. Versatile role of heparanase in inflammation. Matrix Biol. 2013; 32(5):23440.10.1016/j.matbio.2013.02.008

17. Wang, L., Brown, J. R., Varki, A., and Esko, J. D. Heparin's antiinflammatory effects require glucosamine 6-0-sulfation and are mediated by blockade of L- and P-selectins. J. Clin. Invest. 2002 110:127-136.10.1172/JCI14996

18. Lu, L., Liu, Q., Zhu, Y., Chan, K. H., Qin, L., Li, Y., et al. Structurebased discovery of Middle East respiratory syndrome coronavirus fusion inhibitor. Nat. Commun.2004; 5:3067.10.1038/ncomms4067

19. Nadir, Y., Brenner, B., Zetser, A. et al. Heparanase induces tissue factor expression in vascular endothelial and cancer cells. J. Thromb. Haemost.2006; 4:2443-2451.10.1111/j.15387836.2006.02212.x

20. Li JP, Kusche-Gullberg M. Heparan sulfate: biosynthesis, structure, and function. Int Rev Cell Mol Biol. 2016; 325:21573.10.1016/bs.ircmb.2016.02.009

21. Cagno V, Tseligka ED, Jones ST, Tapparel C. Heparansulfate proteoglycans and viral attachment: true receptors or adaptation bias? Viruses. 2019; 11(7):11.10.3390/v11070596

22. Xu, D., and Esko, J. D. Demystifying heparansulfateprotein interactions. Annu. Rev. Biochem. 2014; 83:129157.10.1146/annurev-biochem-060713-035314

23. Gallagher, T. M., and Buchmeier, M. J. Coronavirus spike proteins in viral entry and pathogenesis. Virology.2001; 279:371374.10.1006/viro.2000.0757

24. Shukla, D. and Spear, P.G. Herpesviruses and heparan sulfate: an intimate relationship in aid of viral entry. J. Clin. Invest.2001; 108:503-510.10.1172/JCI13799

25. Lang J, Yang N, Deng J, Liu K, Yang P, Zhang G, Jiang C. Inhibition of SARS pseudovirus cell entry by lactoferrin binding to heparan sulfate proteoglycans. PLoS One. 2011; 6(8):e23710. 10.1371/journal.pone.0023710

26. Nyberg K, Ekblad M, Bergström T, Freeman C, Parish CR, Ferro V, Trybala E. The low molecular weight heparan sulfate-mimetic, PI-88, inhibits cell-to-cell spread of herpes simplex virus. Antiviral Res. 2004; 63(1):15-24 10.1016/j.antiviral.2004.01.001

27. Mercer, J.; Schelhaas, M.; Helenius, A. Virus entry by endocytosis Annu. Rev. Biochem. 2010; 79:803-833. 10.1146/annurevbiochem-060208-104626

28. Somiya, M.; Liu, Q.; Yoshimoto, N.; Iijima, M.; Tatematsu, K.; Nakai, T.; Okajima, T.; Kuroki, K.; Ueda, K.; Kuroda, S. Cellular uptake of hepatitis B virus envelope $\mathrm{L}$ particles is independent of sodium taurocholate cotransporting polypeptide, but dependent on heparan sulfate proteoglycan. Virology .2016; 497:23-32. 10.1016/j.virol.2016.06.024

29. Barth, H., Schafer, C., Adah, M.I. et al.Cellular binding of hepatitis C virus envelope glycoprotein E2 requires cell surface heparan sulfate. J. Biol. Chem. 2003; 278:41003-41012. 10.1074/jbc.M302267200

30. Volz, E.; Hill, V.; McCrone, J.T.; Price, A.; Jorgensen, D.; O’Toole, Á.; Southgate, J.; Johnson, R.; Jackson, B.; Nascimento, F.F.; et al Evaluating the effects of SARS-CoV-2 spike mutation D614G on transmissibility and pathogenicity. Cell. 2021; 184:64-75. 10.1016/j.cell.2020.11.020

31. Dogra, P., Martin, E.B., Williams, A. et al. Novelheparan sulfatebinding peptides for blocking herpesvirus entry. PLoS ONE.2015; 10;e0126239. 10.1371/journal.pone.0126239

32. Jaishankar, D., Yakoub, A.M., Bogdanov, A., Valyi-Nagy, T. and Shukla, D. Characterization of a proteolytically stable D-peptide that suppressesherpes simplex virus 1 infection: implications for the development of entry-based antiviral therapy. J. Virol.2015; 89:1932-1938. 10.1128/JVI.02979-14

33. Fehr, A. R., and Perlman, S. Coronaviruses: an overview of their replication and pathogenesis. Methods Mol. Biol. 2015; 1282:123. 10.1007/978-1-4939-2438-7_1

34. Su, S.,Wong, G., Shi,W., Liu, J., Lai, A. C. K., Zhou, J., et al. Epidemiology, genetic recombination, and pathogenesis of coronaviruses. Trends Microbiol. 2016; 24:490-502. 10.1016/j.tim.2016.03.003

35. Gomes, P. B., and Dietrich, C. P. Distribution of heparin and other sulfated glycosaminoglycans in vertebrates. Comp. Biochem. Physiol. B.1982; 73:857-863. 10.1016/0305-0491(82)90329-7

36. Mycroft-West, C., Su, D., Elli, S., Li, Y., Guimond, S., Miller, G., et al The 2019 coronavirus (SARS-CoV-2) surface protein (Spike) S1 Receptor Binding Domain undergoes conformational change upon heparin binding. BioRxiv. 2020; 971093 10.1101/2020.02.29.971093

37. Seffer MT, Cottam D, Forni LG, Kielstein JT. Heparin 2.0: A New Approach to the Infection Crisis. Blood Purif. 2021;50(1):28-34. $10.1159 / 000508647$

38. Tang N, Bai H, Chen X, Gong J, Li D, and Sun Z. Anticoagulant treatment is associated with decreased mortality in severe coronavirus disease 2019 patients with coagulopathy. J Thromb Haemost.2020; 18:1094-1099. 10.1111/jth.14817

39. Tavassoly O, Safavi F, Tavassoly I. Heparin-binding Peptides as Novel Therapies to Stop SARS-CoV-2 Cellular Entry and Infection. MolPharmacol. 2020; 98(5):612-619. 10.1124/molpharm.120.000098

40. Herold BC, Gerber SI, Polonsky T, Belval BJ, Shaklee PN, Holme K. Identification of structural features of heparin required for inhibition of herpes simplex virus type 1 binding.Virology. 1995; 206(2):1108-16. 10.1006/viro.1995.1034

41. Daviet, F., Guervilly, C., Baldesi, O., Bernard-Guervilly, F. Pilarczyk, E., Genin, A., et al. Heparin induced thrombocytopenia in severe COVID-19 patients. Circulation 2020; 49015 10.1161/CIRCULATIONAHA.120.049015

42. Lozano, R., and Franco, M. E. Incidence of heparin-induced thrombocytopenia in patients with 2019 coronavirus disease. Med. Clin. 2020:23.

43. Duranteau, J., Taccone, F. S., Verhamme, P., Ageno, W., and Force, E. V. G. T. European guidelines on perioperative venous thromboembolism prophylaxis: intensive care. Eur. J. Anaesthesiol. 2018; 35:142146.10.1097/EJA.0000000000000707

44. Shi, C., Wang, C., Wang, H., Yang, C., Cai, F., Zeng, F., et al. The potential of low molecular weight heparin to mitigate cytokine storm in severe COVID-19 patients: a retrospective cohort study. Clin. Transl. Sci. 2020; 12880.10.1111/cts.12880 